\title{
Comparative Analysis of Heart Rate Variability During Different Phases of Menstrual Cycle in Eumenorrhea \& Dysmenorrhea Subjects
}

\author{
JAYAMALA A.K. ${ }^{1}$, PREETHI B.L. ${ }^{2}$, LATHA R. ${ }^{3}$, BHARATHI C. ${ }^{4}$, SHWETHA B.M. ${ }^{5}$ \\ ${ }^{1}$ Dr. A.K. Jayamala, Assistant Professor, Dept of Physiology, SVMCH \& RC, Puducherry \\ ${ }^{2}$ Dr. B.L. Preethi, Associate Professor, Dept of Physiology, MSRMC \& RC, Bangalore \\ ${ }^{3}$ Dr. R. Latha, Professor, Dept of Physiology, SVMCH \& RC, Puducherry \\ ${ }^{4}$ Dr. C. Bharathi, Assistant Professor, Dept of Physiology, AMCH, Salem \\ ${ }^{5}$ B.M. Shwetha, Continuous Rotatory Residential Intern, MSRMC \& RC, Bangalore
}

E-mail: drjayamala2013@gmail.com

Introduction. The reproductive system of women shows regular cyclic changes that teleologically may be regarded as periodic preparations for fertilization and pregnancy [4]. Painful menstruation otherwise called Dysmenorrhea is a common gynecological problem in females of a reproductive age group. Primary dysmenorrhea has prevalence up to $70 \%$ of young females (3). Severe dysmenorrhea is associated with restriction of activity and absenteeism from school and work \& this affects academic performance \& social activities $[6,15,23]$.

Few studies have reported some degree of autonomic imbalance in primary dysmenorrhea [11, 17]. Autonomic nervous system (ANS) regulates the function of various systems in human body to maintain homeostasis and adaptation to stressful situations [12]. Autonomic nervous system of the intrinsic cardiac pacemakers modulates beat to beat variability in heart's rhythm. Analysis of HRV provides a more sensitive noninvasive measure of cardiac autonomic regulation than catecholamine levels and skin conductance [19]. Autonomic nervous system \& endocrine system interact with each other to alter many physiological functions [15] but the extent to which it is related to dysmenorrhea is not yet understood. In eumenorrhea subjects significantly greater HRV and increased vagal activity in follicular phase and increased sympathetic activity during luteal phase is showed by [16, 17, 21].

Since there is paucity of information on the influence of ANS on the changes in time domain parameters of HRV, this study was intended to compare HRV changes during menstrual, follicular and luteal phases of menstrual cycle in both eumenorrhea and dysmenorrhea groups.

Aim. This study had been planned to compare the autonomic activity among eumenorrhea \& dysmenorrhea subjects.

Materials and methods. A longitudinal observational study was conducted among 60 female volunteers aged between 18 \& 25years in the department of physiology in M.S. Ramaiah Medical College Hospital \& Research centre in Bangalore after obtaining Institutional Ethics Committee clearance before the commencement of the study. The subjects were explained the testing procedure and protocol. Informed consent was obtained prior to participation in this study. A detailed medical and menstrual history was obtained from all participants. Standard anthropometric measurements like height, weight, body mass index (BMI) were recorded.

Inclusion criteria. Females between the age group of 18-25years having regular menstrual cycles (21-35days) were included. Females experiencing dysmenorrhea for the last three months with every menstruation \& who are not on any medication were considered as cases. Females who are not suffering from dysmenorrhea were considered as eumenorrhea subjects.

Exclusion criteria. Females with irregular menstrual cycles, or taking treatment for dysmenorrhea like OCPs were excluded. Females who are diag-

(C) Jayamala A.K., Preethi B.L., Latha R. et al., 2017 
nosed to have secondary dysmenorrhea and any other gynecological problems or any co morbid conditions were excluded.

All subjects were given visual analog scale (VAS), if they had pain score $>3$ they were included as dysmenorrheic subjects $(n=30)$ and females with score $<2$ where included in eumenorrhea group (W. Yu-Jen et al., 2016).

Subjects were asked to abstain from caffeine containing beverages on the day of recording. ECG recording was done during Menstrual phase (MP) (day $1-5)$; follicular phase (FP) (day $6^{\text {th }}-14^{\text {th }}$ ) and Luteal phase (LP) (day $15^{\text {th }}-28^{\text {th }}$ ) [18] of the same menstrual cycle. To avoid potential diurnal variations, subjects were tested (HRV) at the same time of day between 8:00 and 9:00 AM [13] in a quiet room 12 hour postprandial [22]. All subjects were asked to be in the supine position with a normal breathing rate and depth with their eyes closed and in a relaxed state at least $15 \mathrm{~min}$ before data collection. $10 \mathrm{~min}$ ECG of the subject were recorded using PHYSIOPAC- PP4 software (MEDICAID SYSTEMS, Chandigarh). The data was analyzed using RMS Vagus HRV software (RMS, India). The time domain parameters of HRV such as mean heart rate (HR, beats per minute); SDNN (millisecond) - Standard deviation of Normal to Normal RR interval; RMMSD(millisecond) - Standard deviation of square of mean root; PNN50 (\%) - Percentage of Consecutive RR interval whose difference is more than 50 millisecond were measured. HRV analysis was extracted using Kubios HRV analysis software.

Statistical analysis. The data were expressed as mean \pm SD. One way ANOVA was applied to compare the HRV data among the 3 phases of menstrual cycle in cases and controls. To test significance paired and unpaired t test was applied. P-value $<0.05$ was considered statistically significant.

Results. The present study compared the time domain (TD) parameters of HRV in 60 eumenorrhea and dysmenorrhea subjects over a period of 6 months duration.

Table 1

General parameters of subjects in dysmenorrhea \& eumenorrhea group; expressed in mean $\pm \mathrm{SD}$

\begin{tabular}{|l|c|c|c|}
\hline \multicolumn{1}{|c|}{$(\boldsymbol{N}=\mathbf{6 0})$} & Dysmenorrhea $(\boldsymbol{n}=\mathbf{3 0})$ & Eumenorrhea $(\boldsymbol{n}=\mathbf{3 0})$ & P value \\
\hline Age $($ years $)$ & $19.4 \pm 1.65$ & $19.47 \pm 1.63$ & 0.8683 \\
\hline Height $(\mathrm{m})$ & $1.58 \pm 0.02$ & $1.59 \pm 0.06$ & 0.4182 \\
\hline Weight $(\mathrm{kg})$ & $54.9 \pm 4.53$ & $54.93 \pm 4.49$ & 0.1426 \\
\hline BMI $\left(\mathrm{kg} / \mathrm{m}^{2}\right)$ & $21.9 \pm 2.19$ & $21.92 \pm 2.15$ & 0.3745 \\
\hline
\end{tabular}

BMI: Body Mass Index; statistical analysis was done by students unpaired ' $t$ ' test. $p$-value $<0.05$ was considered significant.

Table 1 shows the descriptive statistics of the study population. As shown in the above table both the groups did not differ across their demographic details, all this indicates effective randomization.

Table 2

Time domain parameters in Eumenhorrhea subjects

\begin{tabular}{|l|c|c|c|c|}
\hline \multicolumn{1}{|c|}{ Groups } & Mean HR & SDNN (ms) & RMSSD (ms) & PNN50 (\%) \\
\hline MP & $73.83 \pm 10.42$ & $63.72 \pm 23.26$ & $51.86 \pm 17.9$ & $36.15 \pm 18.27$ \\
\hline FP & $72.77 \pm 11.04$ & $65.34 \pm 21.13$ & $54.64 \pm 16.53$ & $40.11 \pm 16.67$ \\
\hline LP & $72.17 \pm 11.54$ & $65.95 \pm 24.60$ & $49.11 \pm 15.25$ & $39.94 \pm 16.27$ \\
\hline MP Vs FP (p value) & 0.6703 & 0.772 & 0.5783 & 0.3535 \\
\hline FP Vs LP (p value) & 0.83 & 0.9286 & 0.2358 & 0.9645 \\
\hline MP Vs LP (p value) & 0.5862 & 0.7156 & 0.4326 & 0.4344 \\
\hline
\end{tabular}

Data is expressed as mean \pm SD. HR-heart rate; SDNN-Standard deviation of Normal to Normal RR interval; RMMSD-Standard deviation of square of mean root; PNN50-Percentage of Consecutive RR interval whose difference is more than 50 millisecond; statistical analysis was done by one-way ANOVA \& paired t-test. $p$-value $<0.05$ was considered significant. 
Table 2 Shows the TD parameters in eumenorrhea subjects. Mean HR, SDNN, RMMSD, PNN50, they did not show any difference between the different phases of menstrual cycle, indicates that there was no much difference in autonomic activity in normal subjects during different phases of menstrual cycle.

Table 3

Time domain parameters in Dysmenhorrhea subjects

\begin{tabular}{|l|c|c|c|c|}
\hline \multicolumn{1}{|c|}{ Groups } & Mean HR & SDNN (ms) & RMSSD (ms) & PNN50 (\%) \\
\hline MP & $78.7 \pm 14.5$ & $60.97 \pm 14.30$ & $38.98 \pm 14.67$ & $38.95 \pm 18.64$ \\
\hline FP & $76.46 \pm 8.11$ & $74.83 \pm 19.05$ & $61.40 \pm 21.43$ & $53.29 \pm 20.28$ \\
\hline LP & $78.23 \pm 6.28$ & $68.23 \pm 21.41$ & $54.99 \pm 18.18$ & $40.66 \pm 16.5$ \\
\hline MP Vs FP & 0.448 & $0.0011^{* *}$ & $0.0001^{* * *}$ & $0.0047^{* *}$ \\
\hline FP Vs LP & 0.3270 & 0.16 & 0.28 & $0.0097 * *$ \\
\hline MP Vs LP & 0.8554 & 0.2030 & $0.0001^{* * *}$ & 0.7407 \\
\hline
\end{tabular}

Data is expressed as mean \pm SD. HR-heart rate; SDNN-Standard deviation of Normal to Normal RR interval; RMMSD-Standard deviation of square of mean root; PNN50-Percentage of Consecutive RR interval whose difference is more than 50 millisecond; statistical analysis was done by one-way ANOVA \& paired t-test. p-value $<0.05^{*},<0.001^{* *},<0.0001 * * *$ was considered significant.

Table 3 Shows that the mean HR is higher in MP when compared with FP \& LP but it is not statistically significant. SDNN, RMMSD, PNN50 have similar behavior, which reflects parasympathetic activity. As shown in table 3, these 3 parameters showed significant difference between MP\& FP in cases. This shows increased parasympathetic activity during follicular phase. No statistical difference is seen between FP \& LP parameters. SDNN, PNN50, RMMSD are less in cases than controls during MP indicating greater sympathetic activity in dysmenorrhea subjects.

Table 4

Time domain parameters in dysmenorrhea subjects vs Eumenhorrhea subjects

\begin{tabular}{|c|c|c|c|c|}
\hline Groups & $\begin{array}{c}\text { Mean HR (p } \\
\text { value) }\end{array}$ & SDNN (p value) & $\begin{array}{c}\text { RMSSD (p } \\
\text { value) }\end{array}$ & PNN50 (p value) \\
\hline MPD Vs MPE & 0.2043 & 0.5827 & $0.0035^{*}$ & 0.2137 \\
\hline FPD Vs FPE & 0.1444 & $0.07^{*}$ & $0.007^{*}$ & $0.0079^{*}$ \\
\hline LPD Vs LPE & $0.0142^{*}$ & 0.7037 & 0.1803 & 0.8663 \\
\hline
\end{tabular}

$\mathbf{M P}_{\mathbf{D}}$ - Menstrual Phase in Dysmenorrhea subjects; $\mathbf{M} \mathbf{P}_{\mathbf{E}}$ - Menstrual Phase in Eumenorrhea subjects. Data is expressed in p-value. HR-heart rate; SDNN-Standard deviation of Normal to Normal RR interval; RMMSD-Standard deviation of square of mean root; PNN50-Percentage of Consecutive RR interval whose difference is more than 50 millisecond; statistical analysis was done by unpaired t-test. p-value $<0.05^{*}$ was considered significant.

In the above table 4 mean HR showed significant difference between 2 groups in luteal phase. It is higher in dysmenorrhea subjects than controls. SDNN, RMSSD are higher in dysmenorrhea subjects in MP\& FP.

Discussion. In the present study, different phases of menstrual cycle in eumenorrhea subjects showed no difference in time domain parameters of HRV. These findings are in agreement with earlier studies where they found no difference in heart rate variability among different phases of menstrual cycle $[13,24]$. A.S. Leicht et al. demonstrated the absence of hormonal influence on HRV during 3 phases of menstrual cycle in eumenorrhea subjects [13]. A.Z.S. Texeira et al. stated that in different phases of menstrual cycle the resting heart rate did not alter in healthy women independently of the use of oral contraceptive pills [20]. 
These results are in contrast with the earlier studies where they found significantly greater HRV in follicular phase and increased sympathetic activity in luteal phase in eumenorrhea subjects $[2,9,21]$. Time domain parameters RMMSD, PNN50 measure short term variation in autonomic activity; in the current study it shows no difference in autonomic activity in normal subjects during different phases of menstrual cycle.

In dysmenorrheal subjects there was a significant difference between menstrual and follicular phase in time domain parameters of HRV. SDNN and RMSSD during menstrual phase showed significant reduction in dysmenorrhea subjects compared to eumenorrhea subjects. This result in dysmenorrhea subjects indicates reduced vagal activity in menstrual phase. Menstrual phase showed reduced HRV and on administration of music to dysmenorrhea subjects showed an increase in parasympathetic activity in menstrual phase [10].

TD parameters of HRV during FP showed significant increase in dysmenorrhea subjects. Time domain parameters indicate parasympathetic activity. Slight reduction in parasympathetic activity was noted during menstrual phase but it was not a statistically significant difference in eumenorrhea subjects. In dysmenorrhea subjects, significant difference was noted in TD parameters between Menstrual \& Follicular phase. In dysmenorrhea subjects TD parameters showed upward trend towards follicular phase, this indicates that compensatory increase in parasympathetic activity. In contrast, another study stated that vasomotor activity regulating sympathetic nervous system activity remains elevated even during the non painful follicular phase in dysmenorrhea subjects [15].

Dysmenorrhea induced pain activates vagus nerve to reduce pain. Physiologically sympathetic \& parasympathetic nervous system functions antagonistically. M.J. Chandler et al. have reported that stimulation of cervical, thoraxic \& cardiac vagal afferents reduces nociceptive neuronal activites [7]. In this study increased vagal activity induced by dysmenorrhea in menstrual phase persists to alter follicular phase as well. Estrogen activates choline acteyltransferase thereby acting as cholinergic agonist by increasing acetylcholine synthesis [5]. Progesterone acts adrenergic agonist by enhancing release of norepinephrine [14].

Females in reproductive age group experience varying symptoms like cramps (dysmenorrhea), food cravings, irritability, depression, breast tenderness, anxiety and mood swings. These symptoms are collectively called Premenstrual syndrome (PMS) [8]. Study by F.C. Baker et al. showed significant reduction in parasympathetic activity during sleep in women with severe PMS in the late- luteal phase of menstrual cycle [1].

Limitations. One limitation could be that we studied HRV indexes only 3 times across the menstrual cycle, once each time in Menstrual phase (MP) (day 1-5); follicular phase(FP) (day 11-21) and luteal phase(LP) (day 21-34), it is better to assess HRV parameters more frequently throughout the menstrual cycle. Second limitation is estimation of endogenous hormones like FSH, LH, estrogen \& progesterone, thyroid hormones, Leptin will give a better understanding of influence of menstrual cycle on autonomic nervous system was not done in our study.

Future studies should be undertaken to address these limitations using a larger sample size.

Conclusion. Women with dysmenorrhea experiences severe menstrual pain due to autonomic imbalance. Young women suffering from dysmenorrhea had reduced parasympathetic activity in menstrual phase but showed a increased parasympathetic activity in follicular phase. This suggests the complex physiological homeostatic mechanisms playing a role to maintain autonomic balance even when the autonomic disturbances occur. 


\section{REFERENCES}

1. Backer FC, Colrain IM, Trinder J. Reduced parasympathetic activity during sleep in the symptomatic phase of severe premenstrual syndrome. J. psychosom Res. 2008;65(1):13-22. 2. Bai $X$, Li J, Zhou L, Li X. Influence Of The Menstrual Cycle On Nonlinear Properties Of Heart Rate Variability In Young Women. American Journal of Physiology - Heart and Circulatory Physiology. 2009;66(2):765-774. 3. Balbi C, Musone R, Menditto A, Prisco LD, Cassese E, D Ajello $M$, Ambrosio $D$. Influence of menstrual factors and dietary habits on menstrual pain in adolescence age. Eur J. Obstet. Gynecol. Reprod. Biol. 2000;91:143-148. 4. Barrett KE, Brooks H, Boitano S, Barman SM. Reproductive Development and [1] Function of the Female Reproductive System. In: 24th edition, Ganong's Review of Medical Physiology. New Delhi: McGraw Hill; 2012; 391-418. 5. Bernardi F, Genazzani AR. The brain: target and source for sex Steroid hormones. In: Women's Health and Menopause, edited by Paoletti R, Crosignani PG, Kenemans P, Wenger NK, Jackson AS. Dordrecht, The Netherlands: Springer, 1999; 137-143. 6. Burnett MA, Antao V, Black A, Feldman K, Grenville A, Lea R et al. Prevalence of primary dysmenorrhea in Canada. J. Obstet Gynecol. Can. 2005;27:765-770. 7. Chandler MJ, Hobbs SF, Bolser DC, Foreman $R D$. Effects of vagal afferent stimulation on cervical spinothalamic tract neurons in monkeys. Pain. 1991;44:81-87. 8. Dickerson M, Mazyck J, Hunter H. Premenstrual syndrome. Am Fam. Phys. 2003;67:1743-1752. 9. Hegzi M, Nasrat H. HRV in young healthy females with primary dysmenorrhea. Bull. Alex. Fac. Med. 2007;43(3):689. 10. Jayamala AK, Preethi BL, Shwetha BM, Bharathi C. Effect of music therapy in dysmenorrhea subjects during menstrual phase of menstrual cycle. International Journal of biomedical research. 2014;05(12):748-750. 11. Kim E, Cho JH, Jung WS, Lee S, Pak SC. Effect of acupuncture on heart rate variability in primary dysmenorrheic women. Am J. Chin. Med. 2011;39:243-249. 12. Latha R, Srikanth $S$, Sairaman H, Dity NRE. Effect of music on heart rate variability and stress in medical students. International Journal of Clinical and Experimental Physiology.2014;01(2):131-134. 13. Leicht AS, Hirning DA, Allen GD. Heart rate variability and endogenous sex hormones during the menstrual cycle in young women. Exp. Physiol. 2003;88:441-446. 14. Mercuro G, Pitzalis L, Podda A, Zoncu S, Pilia I, Melis GB, Cherchi A. Effects of acute administration of natural progesterone on peripheral vascular responsiveness in healthy postmenopausal women. Am J. Cardiol. 1999;84:214-218. 15. Park MK, Watanuki S. Specific physiological responses in women with severe primary dysmenorrhea during the menstrual cycle. Journal of Physiological Anthropology and Applied Human Science. 2005;24;601-609. 16. Sato N, Miyake S, Akatsu J, Kumashiro $M$. Power spectral analysis of heart rate variability in healthy young women during the normal menstrual cycle. Psychosom Med. 1995;57:331-335. 17. Singh K, Srivastava D, Misra AR, Tyagi $M$. Cardiac autonomic activity in young females with primary dysmenorrhea. Indian Journal of Physiology and Pharmacology. 2013;57:246-254. 18. Sneha BS, Sheila RP, Nayantara AK, Balachandra AS. Comparision of cardiac autonomic activity and BMI in different phases of menstrual cycle using heart rate variability. International journal of biomedical and advance research. 2011;02(10):402-409. 19. Task Force of the European Society of Cardiology and the North American Society of Pacing and Electrophysiology. Heart rate variability: Standards of measurement, physiological interpretation, and clinical use. European Heart Journal. 1996;17:354-381. 20. Teixeira ALS, Júnior WF, Moraes EM, Alves HB, Damasceno $V$, Dias MR. Effects of Menstrual Cycle Phase on Resting Heart Rate in Healthy Women. Journal of Exercise Physiologyonline. 2012;15(4):47-54. 21. Tejinder KB, Singh KD, Avnish $K$. Effect of Different Phases of Menstrual Cycle on Heart Rate Variability (HRV). Journal of Clinical and Diagnostic Research. 2015;9(10):CC01-CC04. 22. Widerlov E, Jostel K-G, Claesson $L$, Odlind B, Keisu $M$, Freyschuss $U$. influence of food intake on electrocardiograms of healthy male volunteers. Eur. J. Clin. Pharmacol. 1999;55:619-624. 23. Wilson C, Keye W. A survey of adolescent dysmenorrhea and premenstrual symptom frequency. J. Adolesc. Health. Care. 1989;10:317-322. 24. Yildirir A, Kabakci G, Akgul E, Tokgozoglu L, Oto A. Effects of menstrual cycle on cardiac autonomic innervation as assessed by heart rate variability. Ann Noninv Electrocardiol. 2002;7:60-63. 25. Yu Jen W, Yi Zen W, Mei Ling Y. A prospective comparision study of heat rate variability during menses in young women with dysmenorrhea. Biological Research for Nursing. 2016,18(4):465-472. 


\title{
RESEARCH ARTICLE
}

\section{Comparative Analysis of Heart Rate Variability During Different Phases of Menstrual Cycle in Eumenorrhea \& Dysmenorrhea Subjects}

\author{
A.K. JAYAMALA', B.L. PREETHI \\ R. LATHA ${ }^{3}$, C. BHARATHI ${ }^{4}$, B.M. SHWETHA
}

${ }^{1}$ Dr. A.K. Jayamala, Assistant Professor, Dept of Physiology, SVMCH \& RC, Puducherry

${ }^{2}$ Dr. B.L. Preethi, Associate Professor, Dept of Physiology, MSRMC \& RC, Bangalore

${ }^{3}$ Dr. R. Latha, Professor, Dept of Physiology, SVMCH \& RC, Puducherry

${ }^{4}$ Dr. C. Bharathi, Assistant Professor, Dept of Physiology, AMCH, Salem

${ }^{5}$ B.M. Shwetha, Continuous Rotatory Residential Intern, MSRMC \& RC, Bangalore

Heart rate variability among eumenorrhea \& dysmenorrhea subjects was compared. Different phases of menstrual cycle in eumenorrhea subjects showed no difference in time domain parameters of HRV. In dysmenorrhea subjects there was a significant increase in parasympathetic activity in follicular phase compared to menstrual phase. This suggests the complex physiological homeostatic mechanisms plays a role to maintain autonomic balance even when the autonomic disturbances occur.

Key words: menstrual cycle, eumenorrhea, dysmenorrhea, Heart rate variability. 